\title{
Crack Induced Self-Assembled Grooves for Liquid Crystal Molecules Alignment
}

\author{
TZU-CHIEH LIN, ${ }^{1}$ LI-CHEN HUANG, ${ }^{1}$ \\ TSU-RUEY CHOU, ${ }^{1}$ AND CHIH-YU CHAO ${ }^{1,2}$ \\ ${ }^{1}$ Department of Physics, National Taiwan University, Taipei, Taiwan \\ ${ }^{2}$ Institute of Applied Physics, National Taiwan University, \\ Taipei, Taiwan
}

\begin{abstract}
In this paper, we employ an express and high-throughput method fabricating microand nano-grooves for liquid crystal (LC) molecules alignment. Splitting the polymer film triggers the crack front vibrating back and forth between the two substrates. The propagating wave front brings out self-assembled polymer grooves extending over a large area. The period of the crack induced self-assembled grooves is tunable which consists with the theoretical prediction. This crack-induced grooving (CIG) method can avoid the drawbacks caused by traditional rubbing and provide anchoring energy comparable to that using polyimide rubbing. This novel CIG method offers an appealing alternative to existing technologies for LCs alignment.
\end{abstract}

Keywords Liquid crystals alignment; nanogrooves; self-assembly

\section{Introduction}

Aligning LC molecules is a very important issue in manufacturing LC displays (LCD) and devices. It is well-understood that LC molecules would tend to orient along the groove's direction due to the minimization of LC elastic energy on groove surface [1]. Although traditionally polyimide (PI) rubbing is the most common technique for creating grooves on polymer surface and in turn renders LCs to align parallel to the groove's direction, it still has many intrinsic disadvantages like high-temperature process $\left(\sim 250^{\circ} \mathrm{C}\right)$, dust contaminations, electrostatic problem, and many ions caused by rubbing. E-beam lithography and AFM nano-rubbing were also utilized to tailor the polymer surface with the nano-grooves for LC alignment, but the region of the pattern is very small and these techniques show a very low throughput [2,3]. Besides that, nanoimprint lithography (NIL) and photolithography (PL) were employed to transfer grooves on polymer thin film for aligning LC molecules [4,5]. However, both NIL and PL need a prefabricated mask and the period of grooves is also limited.

Address correspondence to Tzu-Chieh Lin, Department of Physics, National Taiwan University, Taipei 10617, Taiwan. Tel.: +886-2-3366-5197; Fax: +886-2-3366-5088; E-mail: tclin@phys.ntu.edu.tw 
In this paper, we propose a straightforward method splitting the polymer film sandwiched by two substrates for fabricating micro- or nano-grooves to align LC molecules. The scheme of this CIG method is shown in Figure 1 which comprises several steps. In our experiment, strong adhesion between polymer and substrates is influential in forming grooves, but do not affect the period of grooves. As shown in Figure 1(a), pressing and heating the sandwiched polymer between two substrates improve the adhesion. Pull apart the two substrates with a tensile to trigger the crack which vibrates back and forth between two substrates so as to relieve the largest tensile stress in its path [6]. (see Figs. 1(b) and 1(c)) The crack front propagates from one edge to the other to bring out self-formation grooves. Figure 1(d) demonstrates the schematic of LC molecules mainly aligning parallel to the grooves.

In our experiments, we found that the wavelength of periodic grooves is around four times the initial thickness of the thin film which is consistent with that of apreviously published report [7]. Our smallest period is $150 \mathrm{~nm}$ and this CIG method potentially provides the possibility of reaching a smaller period by reducing the film thickness. In this work, the period is tuned for changing anchoring energy to improve the contrast ratio (CR) and the measured opto-electrical curve is comparable to that of traditionally reflective LC cells. Additionally, the anchoring energy of the self-assembled grooves is $\sim 10^{-5} \mathrm{~N} / \mathrm{m}$ which is quite close to the value using PI rubbing. This CIG method generating grooves from a featureless film is low-cost and simple-processing. Moreover, it provides a much more express and largerdomain patterning method than E-beam and AFM lithography. Compare to conventional cloth rubbing and AFM scratching, this CIG method inducing self-assembled grooves also circumvents grazing damage, dust contamination and residual static electricity problems. Especially for LC devices with plastic substrates, it performs a much lower processing temperature which is incapable by PI rubbing. The CIG method not only offers an appealing alternative to existing technologies for

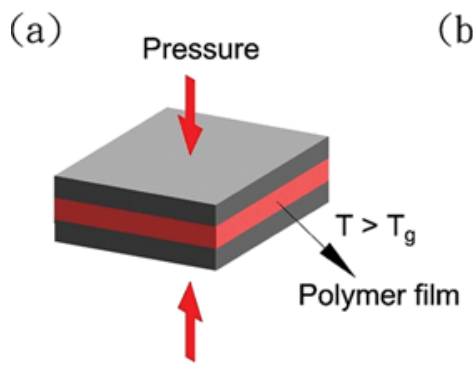

(b)

(c)

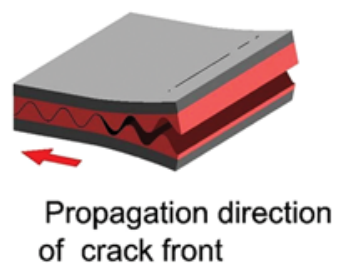

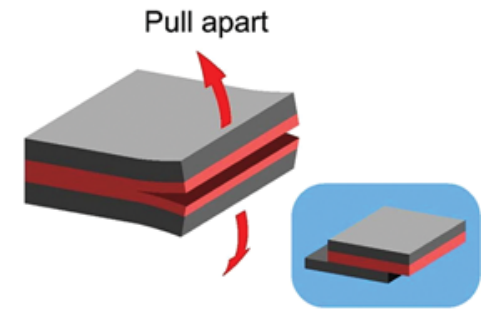

(d)
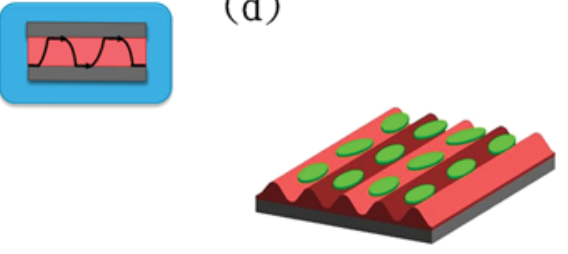

Figure 1. Schematic of the self-assembled grooves formation using the CIG method. (Figure appears in color online.) 
LC molecules alignment but also holds promises to be applied to the roll-to-roll process in the future.

\section{Experiments and Discussions}

\subsection{Sample Preparation}

For the CIG method, amorphous polymer consisting of glassy material is essential to the formation of self-assembled grooves. During the cracking, amorphous polymer, which is non-crystalline and has no-long range molecular order, would create grooves with the same shape across a large area. On the contrary, polycrystalline material would hamper self-assembled grooves to form owing to its anisotropic property. In our experiments, we choose polystyrene (PS, with a molecular weight of $\mathrm{M}_{\mathrm{w}}=1.5 \mathrm{~kg} / \mathrm{mol}$ ) which is linear homopolymer to be dissolved in the toluene solvent $(0.5-2 \%$ solution by weight). During spin coating, the volatile toluene solvent would evaporate. Baking the substrates a little over the toluene's boiling point $\left(T_{b}=110\right.$ ${ }^{\circ} \mathrm{C}$ ) could facilitate to remove the solvent. After casting uniform polymer film on a silicon substrate, we overlaid this substrate with the other bare substrate. Apply pressure and heat the whole sandwich structure at temperature $\mathrm{T}=140{ }^{\circ} \mathrm{C}$ simultaneously. The temperature a little higher than the glass transition temperature of PS $\left(\mathrm{T}_{\mathrm{g}}=115^{\circ} \mathrm{C}\right)$ could change glassy PS film into rubbery. Pressing and heating cause polymer film to attach more strongly to the substrate. If the adhesion is not enough, the separation through cracking would occur in the interface between the PS film and silicon surface, not within the PS film, then grooves would not form. Increasing adhesive force would extend the area of pattern of the grating for several centimeters or even larger. The applied heat and pressure were released after $10 \mathrm{~min}-$ utes. The important point is that sample separation must be at the temperature below $\mathrm{T}_{\mathrm{g}}$ of polymer. If the separation temperature was higher than $\mathrm{T}_{\mathrm{g}}$, self-assembled grooves would not appear due to the rubbery polymer film which is easily deformed. After being cooled down to room temperature $\left(\sim 25^{\circ} \mathrm{C}\right)$, PS film become brittle and the sample was fractured into two pieces to result in the formation of polymer grooves with the complementary shape on each substrate. Figure 2 shows the optical micrograph of CIG grating (period $\sim 800 \mathrm{~nm}$ ) across a large area.

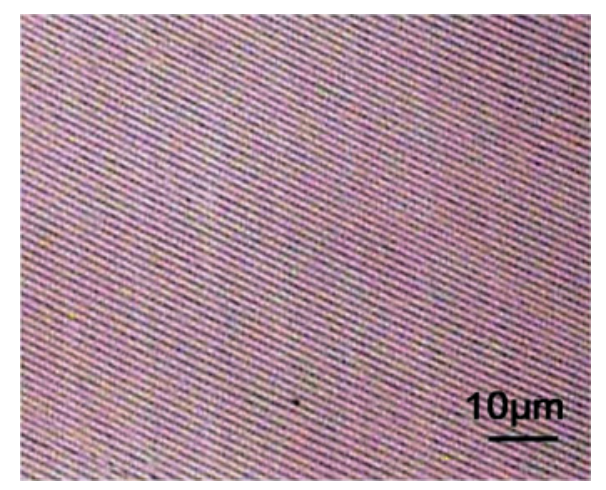

Figure 2. Optical micrograph of self-assembled grooves with period $\sim 800 \mathrm{~nm}$ from a $200 \mathrm{~nm}$ thickness PS film after using the CIG method. (Figure appears in color online.) 
In the following, we measure the details of self-assembled grooves by AFM and scanning electron microscope (SEM).

\subsection{Measurements of Self-Assembled Polymer Grooves}

Varying the concentration of PS toluene solution, from $0.5 \%$ to $2 \%$ could get different PS film thickness after casting at the fixed frequency. More dilute PS solution would result in a thinner film. In our work, the smallest film thickness is $35 \mathrm{~nm}$ from $0.5 \%$ PS toluene solution. By increasing the concentration of solution to $2 \%$, the film thickness comes up to around $200 \mathrm{~nm}$. Due to the sample arrangement is a little staggered, we could easily split the attached sample apart into two substrates both with polymer grooves on the surfaces. The grooves on bottom and upper silicon substrates are nonsymmetrical, but complementary and with the same period. Figure 3 is the complementary grooves with period $\sim 600 \mathrm{~nm}$ on the each substrate. Inspect the morphology of PS film by AFM, there are different period and height of grooves from different film thickness (see Figure 4). With regards to Figure 4, when the polymer thickness are 150,180, and $190 \mathrm{~nm}$, the pitch of grooves are about 590,732, and $755 \mathrm{~nm}$, and the height are 35,100 , and $80 \mathrm{~nm}$ respectively. The height is not exactly equal to the film thickness. It depends on the path of the vibrating crack front and is mostly around the half of thickness or even smaller. In this work, we observe that adhesion or annealing temperature would not affect the period of grooves, while it merely determines whethergrooves would form or not. We found the period $p$ of self-assembled grooves solely depends on the film thickness $d$ and the period is around four times the thickness which is in consistent with Chou's work [7,8]. Hence the period could simply vary by adequately tuning the film thickness.

Figure 5 represents SEM photographs of PS grooves of four different periods. Before taking SEM photographs, sputter gold thin film on PS surface is needed for preventing PS to melt at a local high energy. From Figure 5(a) to (c), the period is about 792, 895, $980 \mathrm{~nm}$ formed in film thickness of 200, 220, $240 \mathrm{~nm}$ respectively. Figure 5(d) demonstrates self-assembled groove across a large area $(p \sim 900 \mathrm{~nm})$.

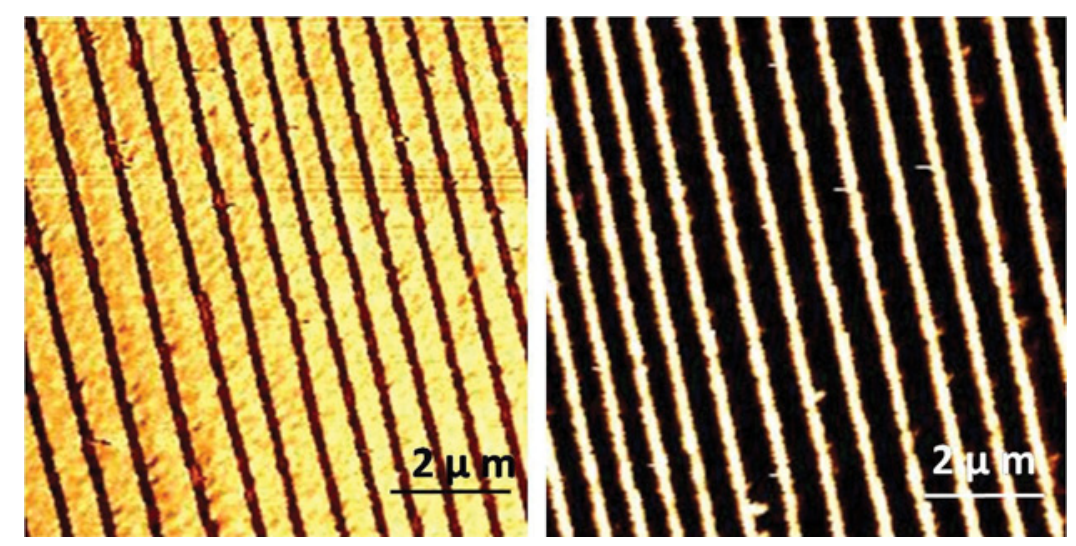

Figure 3. The two AFM images are surface morphology of polymer grooves from the upper and bottom substrates of the same sample after splitting. The grooves on each substrate are nonsymmetrical but complementary and with the same period $(\sim 600 \mathrm{~nm})$. (Figure appears in color online.) 


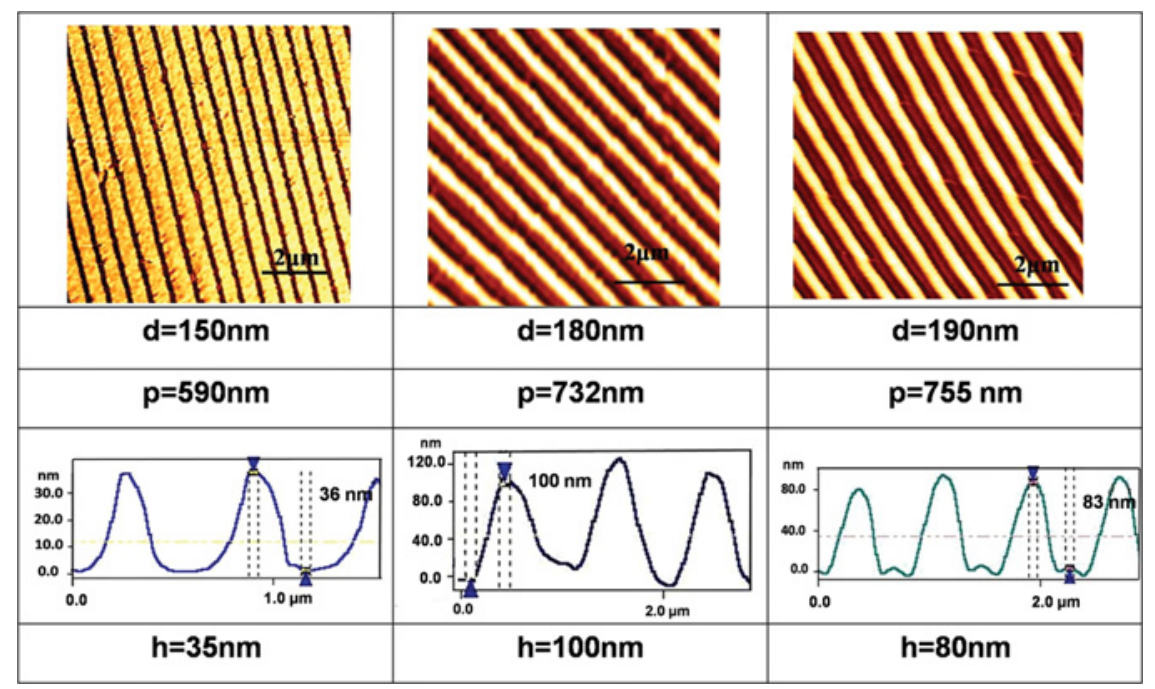

Figure 4. AFM measurement of the period and height of some self-assembled grooves. (Figure appears in color online.)

According to the results using both AFM and SEM, we find the grooves period $p$ is as a function of film thickness $d$. Figure 6 demonstrates the relationship between period $p$ and thickness $d$. By varying the PS concentration as mentioned above, the obtained film thickness is ranging from $35 \mathrm{~nm}$ to $245 \mathrm{~nm}$. We measured the period $p$ of grooves is from $150 \mathrm{~nm}$ to $960 \mathrm{~nm}$. However, for enough alignment in LC devices, the period of grooves should be controlled below the LC correlation length $(\sim 1 \mu \mathrm{m})$ [9].

\subsection{Fabrication and Measurements of Liquid Crystal Cells}

We utilized a silicon substrate with crack-induced polymer grooves as the bottom substrate which was then placed on a conventional indium-tin-oxide (ITO) glass coated with PI planar alignment layer as the upper substrate. The direction of crack-induced grooves was set orthogonal to the PI rubbing direction on the

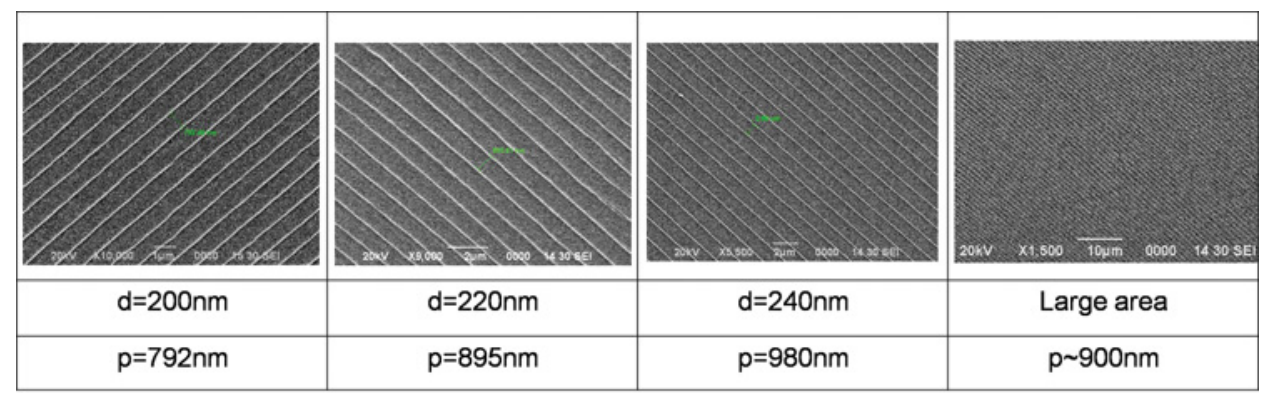

(a)

(b)

(c)

(d)

Figure 5. SEM micrographs of the self-assembled grooves with different periods (PS film is coated with gold film). (Figure appears in color online.) 


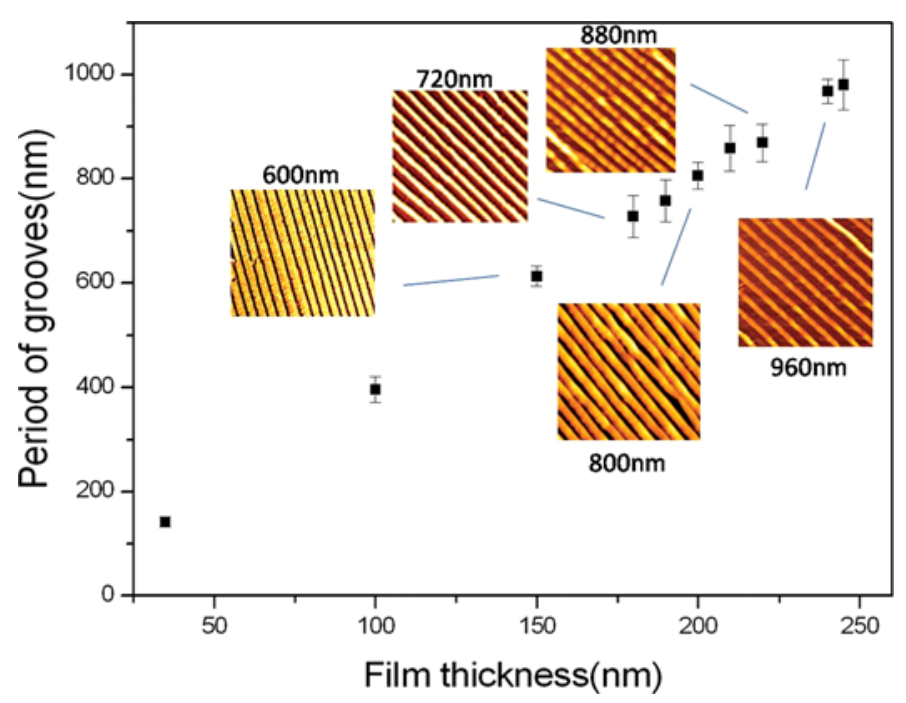

Figure 6. Period of grooves as a function of polymer film thickness. (The insets are AFM images and the width of each inset is $10 \mu \mathrm{m}$.). (Figure appears in color online.)

opposite substrate. Then, we filled this empty cell with a 4-n-pentyl-4'-cyanobiphenyl (5CB) LCs at room temperature and the cell gap is $1.5 \mu \mathrm{m}$. Therefore, the director configuration of LCs sandwiched between the crack-induced grooving substrate and rubbed PI layer becomes $90^{\circ}$ twisted. The cells are inspected by a polarizing optical microscope in its reflection mode and the polarizer setting is demanded to obtain maximum darkness with zero voltage for the LC cell (see Fig. 7(a)). Thus, the patterned area (period $\sim 800 \mathrm{~nm}$, height $\sim 100 \mathrm{~nm}$ ) of the cell belongs to normal black. The polarizer setting makes the polarizer and the LC director near the upper substrate not parallel but with some angle to form a mixed-mode twisted nematic (MTN) LC cell [10]. In direct-view display, the MTN LC cell requiring only one front polarizer could thus prevent the parallax. The doped and polished silicon substrate patterned by crack-induced self-assembled grooves could thus be simultaneously used for LC alignment, the cell electrode, and the mirror reflector.

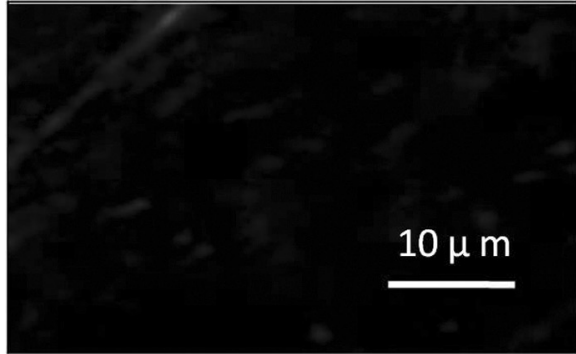

(a)

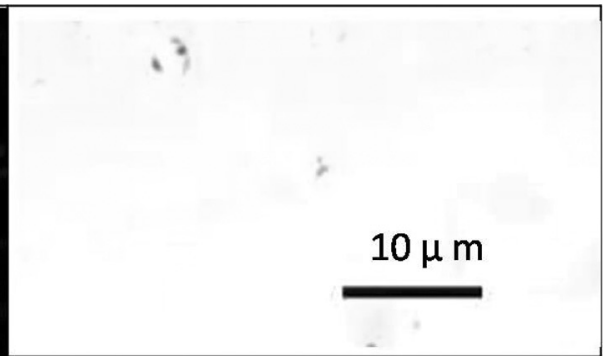

(b)

Figure 7. The crack-induced self-assembled PS grooves are employed to align LC molecules and used to fabricate LC cells as MTN cells. Under polarizing optical microscope, (a) is the NB state with zero applied voltage and (b) is the bright state with bias voltage $\sim 6 \mathrm{~V}$. 
(a)

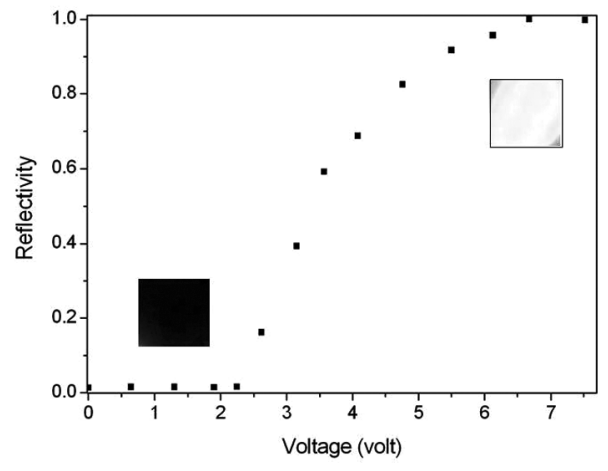

(b)

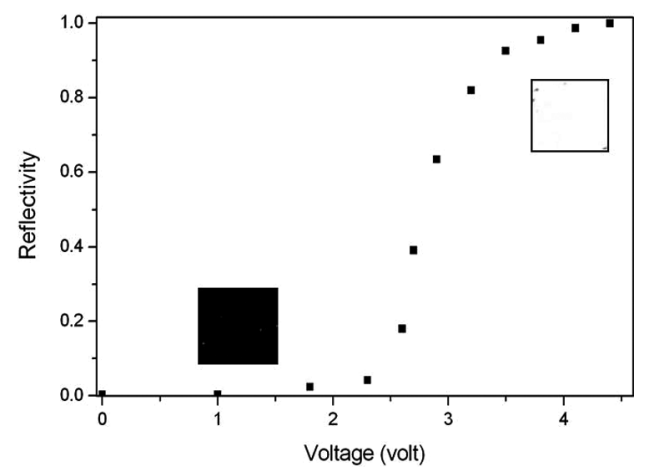

Figure 8. Reflectivity of MTN cells with different groove periods. (The insets are micrographs under polarizing optical microscope.)

Applying the voltage between the ITO and p-type silicon substrates, the patterned LC cell, i.e., normally black mode, started to change its phase retardation and became brighter as shown in Figure 7(b).

Figure 8 demonstrates the voltage-dependent light reflectance (RV curve) of the reflective MTN LC cell. Compare Figure 8(a) and (b), the threshold voltages of these two RV curves are both around $2.3 \mathrm{~V}$, because the threshold voltage is mainly determined by the LC material [11]. Increase the applied voltage, the phase retardation came into $\pi$ resulting in the cell being the bright state. According to Berreman's theory, the anchoring energy resulted from grooves is $\mathrm{W}=2 \pi^{3} \mathrm{~K} \mathrm{~A}^{2} / \lambda^{3}[12]$, which $\mathrm{A}$ is the amplitude, $\lambda$ is the period of groove, and $\mathrm{K}$ is the Frank elastic constant of the LC $\left(\mathrm{K}_{5 \mathrm{CB}}=2 \times 10^{-12} \mathrm{~N}\right)$. For a MTN LC cell in Figure 8(a), with $\lambda \sim 800 \mathrm{~nm}$ and $\mathrm{A} \sim 100 \mathrm{~nm}$, the anchoring energy $\mathrm{W}$ is $\sim 2.4 * 10^{-6} \mathrm{~N} / \mathrm{m}$. For the other MTN cell in Figure $8(\mathrm{~b})$, with $\lambda \sim 600 \mathrm{~nm}$ and $\mathrm{A} \sim 85 \mathrm{~nm}$, the anchoring energy $\mathrm{W}$ is $\sim 4.1 * 10^{-6} \mathrm{~N} / \mathrm{m}$ which is $\sim 1.7$ times that in Figure $8(\mathrm{a})$. The larger anchoring energy mooring LC molecules more strongly should improve the dark state and thus achieve a higher contrast ratio (CR) in LC device. In our experiments, we found that the CR of the MTN LC cell in Figure 8(b) is about 350, which is five times larger than that in Figure 8(a) (CR only $\sim 75$ ), and this result coincides with the calculated anchoring energy.

Nowadays, the anchoring energy of conventional PI rubbing is $10^{-4} \sim 10^{-5} \mathrm{~N} / \mathrm{m}$ and for AFM rubbing is $\sim 10^{-6} \mathrm{~N} / \mathrm{m}[13,14]$. As already stated, the anchoring energy of crack-induced polymer grooves is $\sim 10^{-5} \mathrm{~N} / \mathrm{m}$, which is an order of magnitude between PI and AFM rubbing. This anchoring energy of the PS self-assembled grooves is enough to give a good control of LC molecules.

\section{Conclusions}

We utilize an express and high-throughput method for aligning LC molecules. More than successfully aligning LCs, the CIG method generating self-assembled grooves also enables to align other molecules, like dyes and biomolecules, for optoelectronic device and cell adhesion applications, etc. $[15,16]$. In our experiment, the measured period of grooves is found to be proportional to the film thickness. Thus, the period 
could be tuned as required via changing the film thickness. The anchoring energy of the crack-induced grooves is approaching to that of conventional PI rubbing. This cutting edge method is low cost, low process temperature, no dust contamination, no residual static electricity, and holds promise of patterning self-formation grooves on glass or plastic substrates for LCD application. It provides an alternative comparable to the existing mainstream LCD technologies and enables to be applicable to roll-to-roll process in the future.

\section{Acknowledgment}

One of us (CYC) acknowledges the support from the National Science Council and Ministry of Education of the Republic of China.

\section{References}

[1] Frank, F. C. (1958). Discuss. Faraday Soc., 25, 19.

[2] Tennant, D. M., Koch, T. L., Mulgrew, P. P., Gnall, R. P., Ostermeyer, J.-M., \& Verdiell, M. (1992). J. Vac. Sci. Technol., B 10, 2530.

[3] Kim, J. H., Yoneya, M., \& Yokoyama, H. (2002). Nature, 420, 159.

[4] Chou, S. Y., Krauss, P. R., \& Renstrom, P. J. (1996). Science, 272, 85.

[5] Hill, K. O., Malo, B., Bilodeau, F., Jackson, D. C., \& Albert, J. (1993). Appl. Phys. Lett., 62, 1035.

[6] Freund, B. (2007). Nat. Nanotechnol., 2, 537.

[7] Pease III, L. F., Deshpande, P., Wang, Y., Russel, W. B., \& Chou, S. Y. (2007). Nat. Nanotechnol., 2, 545.

[8] Deshpande, P. A. (2005). PhD Dissertation Princeton University.

[9] Rastegar, A., Skarabot, M., Blij, B., \& Th. Rasing, J. (2001). Appl. Phys., 89, 960.

[10] Wu, S. T., et al. (2001). Reflective Liquid Crystal Displays. Lowe, A. C. (Ed.), Wiley-SID: UK, pp. 89-108.

[11] Nie, X., Lu, R., Xianyu, H., Wu, T. X., \& Wu, S. T. (2007). J. Appl. Phys., 101, 103110.

[12] Berreman, D. W. (1972). Phys. Rev. Lett., 28, 1683.

[13] Yokoyama, H. (1988). Mol. Cryst. Liq. Cryst., 165, 265.

[14] Kim, J. H., Yoneya, M., Yamamoto, J., \& Yokoyama, H. (2002). Nanotechnology, 13, 133.

[15] Burn, P. L., Holmes, A. B., Kraft, A., Bradley, D. D. C., Brown, A. R., Friend, R H., \& Gymer, W. (1992). Nature, 356, 47.

[16] Arnold, M., Cavalcanti-Adam, E. A., Glass, R., Blümmel, J., Eck, W., Kantlehner, M., \& Kessler, H., Spatz, J. P. (2004). ChemPhysChem, 5, 383. 
Copyright of Molecular Crystals \& Liquid Crystals is the property of Taylor \& Francis Ltd and its content may not be copied or emailed to multiple sites or posted to a listserv without the copyright holder's express written permission. However, users may print, download, or email articles for individual use. 